\title{
Sequence of Maps Between Hopf and Aharonov-Bohm Bundles
}

\author{
M. Socolovsky \\ Instituto de Ciencias Nucleares, Universidad Nacional Autónoma de México, Circuito Exterior, Ciudad \\ Universitaria, 04510, México D. F., México \\ Email: socolovs@nucleares . unam.mx
}

\begin{abstract}
The existence of the Aharonov-Bohm $(A-B)$ effect with its associated $U(1)$-bundle $\xi_{A-B}$, and the uniqueness -up to homotopy- of a continuous function $S^{2} \rightarrow \mathbb{C}^{*}$, induce a unique map -up to isomorphism- between the Hopf bundles with zero and unit Chern number, respectively $\xi_{0}: S^{1} \rightarrow S^{2} \times S^{1} \rightarrow S^{2}$ and $\xi_{1}: S^{1} \rightarrow S^{3} \rightarrow S^{2}$. This establishes a tight relation between $\xi_{0}$ and $\xi_{1}$ through $\xi_{A-B}$, and therefore between the $A-B$ effect and the hypothetical unit magnetic charge when the Dirac connection in $\xi_{1}$ is considered.
\end{abstract}

Keywords: Aharonov-Bohm effect, Hopf bundles, magnetic charge

\section{Introduction}

In a recent paper [1] we showed that, if $\xi_{A-B}: S^{1} \rightarrow\left(T_{0}^{2}\right)^{*} \stackrel{p r_{1}}{\longrightarrow}\left(D_{0}^{2}\right)^{*}$ and $\xi_{D} \equiv \xi_{1}: S^{1} \rightarrow S^{3} \stackrel{\pi_{D}}{\longrightarrow} S^{2}$ are the $U(1)$-bundles where the Aharonov-Bohm $(A-B)[2]$ effect and the unit Dirac monopole $(D)[3,4]$ are represented, then $\xi_{A-B}$ turns out to be isomorphic to the pull-back of $\xi_{D}$ induced by the inclusion of the corresponding base spaces: $\iota: \mathbb{C}^{*} \rightarrow S^{2}$. $\left(S^{1}=U(1), S^{2} \cong \mathbb{C} \cup\{\infty\},\left(D_{0}^{2}\right)^{*} \cong \mathbb{R}^{2^{*}} \cong \mathbb{C}^{*}\right.$ is the punctured open 2-disk, $\left(T_{0}^{2}\right)^{*}=\left(D_{0}^{2}\right)^{*} \times S^{1}$ is the open solid 2-torus minus a circle, $S^{k}, k=1,2,3$, are spheres, $p r_{1}$ is the projection in the first entry, and $\pi_{D}$ is the Hopf map $\pi_{D}\left(z_{1}, z_{2}\right)=z_{1} / z_{2}$ if $z_{2} \neq 0$ and $\infty$ if $z_{2}=0$, with $\left\|\left(z_{1}, z_{2}\right)\right\|=1$.) In particular, in the context of bundle theory, this allows to prove through the pull-back of the Dirac connection and the use of the Dirac quantization condition, the well known fact that the $A-B$ effect vanishes when the flux in the solenoid equals an integer times the quantum of magnetic flux $2 \pi \hbar c /|e|$ associated with the electric charge $|e|$. Thus, one obtains a consequence on the observed $A-B$ effect $[5,6]$ from the up to now only hypothetical existence of magnetic charges i.e. a sort of "passage" from $D$ to $A-B$.

An immediate question that arises is if it is possible an "inverse passage": from $A-B$ to $D$, which might, at least from a purely mathematical point of view, reinforce the idea of the real existence of magnetic charges. In this note, we try to come close to a positive answer to this question using the fact that, up to homotopy, the unique continuous map from the base space of $\xi_{D}$ to the base space of $\xi_{A-B}$ is a constant map:

$$
\kappa: S^{2} \rightarrow \mathbb{C}^{*}, b \mapsto \kappa(b)=z_{0}
$$

where $z_{0}$ is an arbitrary (non-zero) complex number.

In section 2. we show that the pull-back of $\xi_{A-B}$ induced by $\kappa$ (or any other function in the homotopy class of $\kappa[7]$ ) is isomorphic to the trivial Hopf bundle $\xi_{0}: S^{1} \rightarrow S^{2} \times S^{1} \stackrel{p r_{1}}{\longrightarrow} S^{2}$ corresponding to zero Chern number, and therefore to zero magnetic charge in the bundle theory of magnetic monopoles. In section 3., through the composition of bundle maps, we obtain a canonical map $\xi_{0} \rightarrow \xi_{1}$, thus establishing a tight relation between $\xi_{0}$, the "sandwiched" bundle $\xi_{A-B}$, and $\xi_{1}$, where the unit magnetic charge is described. Section 4 . is devoted to a conclusion.

\section{Isomorphism Between $\kappa^{*}\left(\xi_{A-B}\right)$ and $\xi_{0}$}

$\kappa$ induces the pull-back bundle $\kappa^{*}\left(\xi_{A-B}\right)$ with total space $P_{\kappa^{*}\left(\xi_{A-B}\right)} \subset S^{2} \times\left(\mathbb{C}^{*} \times S^{1}\right)$ defined by

$$
P_{\kappa^{*}\left(\xi_{A-B}\right)}=\left\{\left(b,\left(z, e^{i \varphi}\right)\right) \mid \kappa \circ p r_{1}=p r_{1} \circ p r_{2}\right\}
$$


i.e. such that the following diagram commutes:

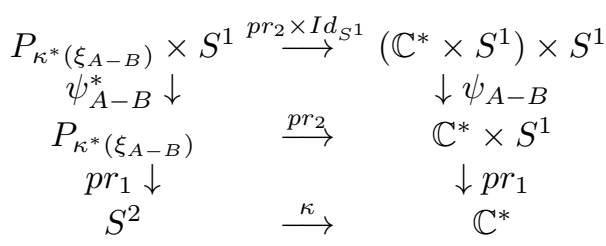

Diagram 1

where $\psi_{A-B}$ and $\psi_{A-B}^{*}$ are the right actions of $S^{1}$ over $P_{\xi_{A-B}} \cong \mathbb{C}^{*} \times S^{1}$ and $P_{\kappa^{*}\left(\xi_{A-B}\right)}$, and $p r_{2}$ is the projection in the second entry. In fact, for the lower part of the diagram one has $\kappa \circ p r_{1}\left(b,\left(z, e^{i \varphi}\right)\right) \kappa(b)=$ $z_{0}=p r_{1} \circ p r_{2}\left(b,\left(z, e^{i \varphi}\right)\right)=p r_{1}\left(z, e^{i \varphi}\right)=z$, and so

$$
P_{\kappa^{*}\left(\xi_{A-B}\right)}=\left\{\left(b,\left(z_{0}, e^{i \varphi}\right)\right), b \in S^{2}, \varphi \in[0,2 \pi)\right\}
$$

while for the upper part of the diagram it holds $\psi_{A-B} \circ\left(p r_{2} \times I d_{S^{1}}\right)=p r_{2} \circ \psi_{A-B}^{*}$ :

$\psi_{A-B} \circ\left(p r_{2} \times I d_{S^{1}}\right)\left(\left(b,\left(z, e^{i \varphi}\right)\right), e^{i \lambda}\right)=\psi_{A-B}\left(p r_{2}\left(\left(b,\left(z, e^{i \varphi}\right)\right), e^{i \lambda}\right)=\psi_{A-B}\left(\left(z, e^{i \varphi}\right), e^{i \lambda}\right)=\left(z, e^{i(\varphi+\lambda)}\right)\right.$, $p r_{2} \circ \psi_{A-B}\left(\left(b,\left(z, e^{i \varphi}\right)\right), e^{i \lambda}\right)=p r_{2}\left(b,\left(z, e^{i(\varphi+\lambda)}\right)\right)=\left(z, e^{i(\varphi+\lambda)}\right)$.

Since $z_{0}$ is a fixed (but otherwise arbitrary) non-zero complex number,

$$
\Phi: P_{\kappa^{*}\left(\xi_{A-B}\right)} \rightarrow S^{2} \times S^{1},\left(b,\left(z_{0}, e^{i \varphi}\right)\right) \mapsto\left(b, e^{i \varphi}\right)
$$

is an homeomorphism, and therefore one has the bundle isomorphism

$$
\xi_{0} \stackrel{\left(I d_{S^{2}}, \Phi^{-1}\right.}{\longrightarrow} \kappa^{*}\left(\xi_{A-B}\right)
$$

at the extreme left of Diagram 2.

Remark: The existence of an isomorphism $\xi_{0} \rightarrow \kappa^{*}\left(\xi_{A-B}\right)$ can be proved from the fact that $\iota \circ \kappa$ is constant and therefore the Chern class of $\kappa^{*}\left(\xi_{A-B}\right)$ is zero.

\section{Bundle Map $\xi_{0} \rightarrow \xi_{D}$}

Putting together the pull-back of $\xi_{D}$ by $\iota$, namely $\iota^{*}\left(\xi_{D}\right)$, and the isomorphism $\xi_{A-B} \stackrel{\left(I d_{\mathbb{C}^{*}, \Psi^{-1}}\right)}{\longrightarrow} \iota^{*}\left(\xi_{D}\right)$, respectively given in sections 4 . and 5 . of Ref. [1], and the results of the previous section, one obtains the sequence of bundle maps

$$
\xi_{0} \stackrel{\cong}{\longrightarrow} \kappa^{*}\left(\xi_{A-B}\right) \longrightarrow \xi_{A-B} \stackrel{\cong}{\longrightarrow} \iota^{*}\left(\xi_{D}\right) \longrightarrow \xi_{D}
$$

represented in detail in the following commuting diagram:

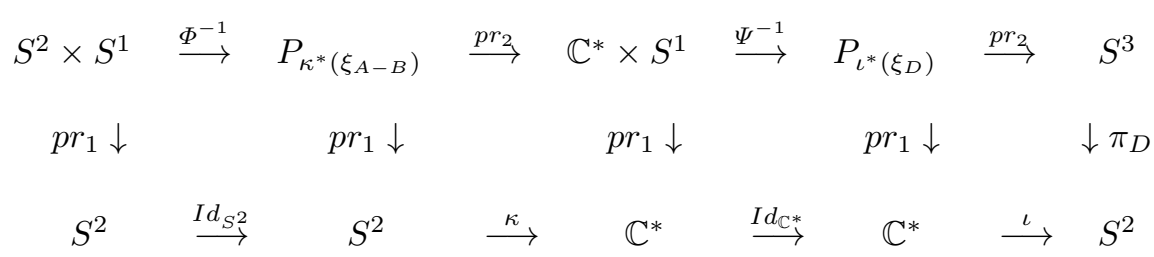

Diagram 2

(For simplicity, we omit the upper part of the diagram, involving the right actions $\psi_{0}, \psi_{A-B}^{*}, \psi_{A-B}, \psi_{D}^{*}$ and $\psi_{D}$ of $S^{1}$ on $S^{2} \times S^{1}, P_{\kappa^{*}\left(\xi_{A-B}\right)}, \mathbb{C}^{*} \times S^{1}, P_{\iota^{*}\left(\xi_{D}\right)}$ and $S^{3}$, respectively.)

Since the composition of bundle maps is a bundle map, one obtains the map between the Hopf bundles $\xi_{0}$ and $\xi_{1}$ :

$$
\xi_{0} \stackrel{(\alpha, \bar{\alpha})}{\longrightarrow} \xi_{1}
$$

where

$$
\alpha=\iota \circ I d_{\mathbb{C}^{*}} \circ \kappa \circ I d_{S^{2}}, \alpha(b)=z_{0}
$$


and

$$
\bar{\alpha}=p r_{2} \circ \Psi^{-1} \circ p r_{2} \circ \Phi^{-1}, \bar{\alpha}\left(b, e^{i \varphi}\right)=\frac{\left(z_{0}, 1\right)}{\left\|\left(z_{0}, 1\right)\right\|} e^{i \varphi}
$$

represented in diagram 3 :

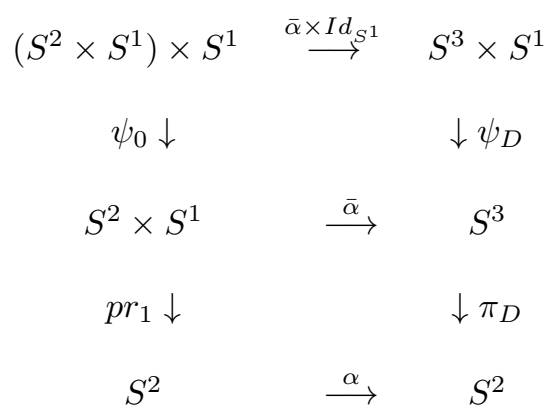

Diagram 3

\section{Conclusion}

Eq. (6) or Diagram 2 clearly exhibit the fact that the bundle corresponding to the $A-B$ effect, $\xi_{A-B}$, is sandwiched between the Hopf bundles corresponding to zero and unit Chern numbers, respectively $\xi_{0}$ and $\xi_{1}$. A remarkable fact of this construction is its uniqueness, in the sense that the inclusion $\iota: \mathbb{C}^{*} \rightarrow S^{2}$ is canonical and the map $\kappa: S^{2} \rightarrow \mathbb{C}^{*}$ is unique up to homotopy (the arbitrariness of the choice of $z_{0} \in \mathbb{C}^{*}$ is irrelevant). This establishes a strong mathematical relation between the $U(1)$-bundles $\xi_{0}, \xi_{A-B}$ and $\xi_{D}$, and therefore between the $A-B$ effect and the Dirac magnetic monopoles when these are described in the language of fiber bundle theory.

Finally, we want to comment that any smooth or continuous map $\alpha: S^{2} \rightarrow S^{2}, \eta \rightarrow \alpha(\eta)$, induces a bundle morphism $\xi_{0} \stackrel{(\alpha, \bar{\alpha})}{\longrightarrow} \xi_{1}$ with $\bar{\alpha}\left(\eta, e^{i \varphi}\right)=\frac{(\alpha(\eta), 1)}{\|(\alpha(\eta), 1)\|} e^{i \varphi}$. This infinite set of morphisms accommodates into an infinite set of homotopy classes indexed by the integers since $\pi_{2}\left(S^{2}\right) \cong \mathbb{Z}$. The morphism $(\alpha, \bar{\alpha})$ given by (8) and (9) is the one induced by the $A-B$ bundle.

Acknowledgments. The author thanks G.L. Naber and J.M. Isidro San Juan for their comments and criticism. Also, to IAFE (UBA-CONICET, Argentina) for hospitality.

\section{References}

1. M. Socolovsky, "Aharonov-Bohm effect, Dirac monopole, and bundle theory," Theoretical Physics (to appear); Vol.3, Nr. 3, 2018.

2. Y. Aharonov and D. Bohm, "Significance of electromagnetic potentials in the quantum theory," Physical Review 15 485-491., 1959.

3. P. Dirac, "Quantized singularities in the electromagnetic field," Proceedings of the Royal Society A133, 60-72., 1948.

4. _— "The theory of magnetic poles," Physical Review 74, 817-830., 1948.

5. R. Chambers, "Shift of an electron interference pattern by enclosed magnetic flux," Physical Review Letters $\mathbf{5}$, 3-5., 1960.

6. M. Peshkin and A. Tonomura, "The Aharonov-Bohm effect," Springer, Berlin., 1989.

7. N. Steenrod, "The topology of fibre bundles," Princeton University Press, N.J., 1951. 